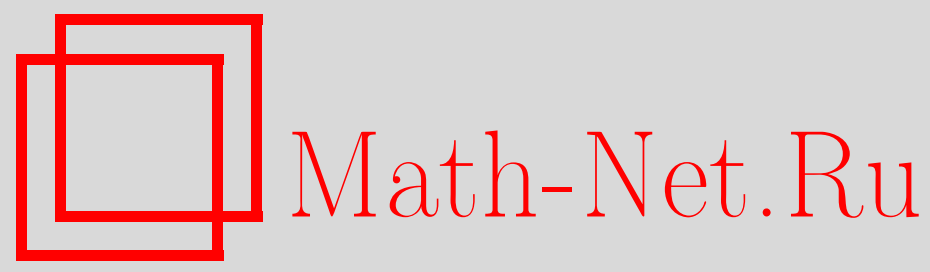

Ю. Ф. Коробейник, Об аналитических решениях проблемы Бореля, Матем. заметки, 2000, том 67, выпуск 4, 525-538

DOI: https://doi.org/10.4213/mzm868

Использование Общероссийского математического портала Math-Net.Ru подразумевает, что вы прочитали и согласны с пользовательским соглашением http://www.mathnet.ru/rus/agreement

Параметры загрузки:

IP: 54.162 .27 .143

26 апреля 2023 г., 15:55:14

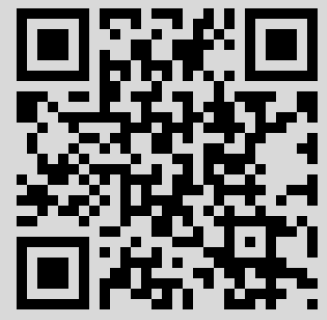


УДК 517.9

\section{ОБ АНАЛИТИЧЕСКИХ РЕШЕНИЯХ ПРОБЛЕМЫ БОРЕЛЯ}

Ю. Ф. Коробейник

Пусть $\mathscr{F}$ - плотное в себе множество в $\mathbb{C}$ с непустой связной внутренностью, содержащее начало координат; $C^{\infty}(\mathscr{F})$ - пространство комплекснозначных функций, бесконечно дифференцируемых на $\mathscr{F}$. Для некоторых классов множеств $\mathscr{F}$ доказывается для произвольной последовательности $\left\{d_{n}\right\}_{n=0}^{\infty}$ комплексных чисел существование функции $f$ из $C^{\infty}(\mathscr{F})$ такой, что $f^{(n)}(0)=d_{n}, n=0,1,2, \ldots$, и исследуется характер ее аналитичности. Функция $f$ строится в виде различных функциональных рядов: степенного, из простейших дробей и из экспонент. Рассматриваются также аналитические решения многомерной задачи Бореля.

Библиография: 10 названий.

1. Пусть $\mathscr{F}$-полное в себе множество в $\mathbb{C}$ со связной (возможно, пустой) внутренностью int $\mathscr{F}$, содержащее начало координат. Комплекснозначная функция $f$, определенная на $\mathscr{F}$, называется дифферениируемой в некоторой точке $z_{0}$ из $\mathscr{F}$, если существует конечньй предел

$$
\lim _{z \rightarrow z_{0}} \frac{f(z)-f\left(z_{0}\right)}{z-z_{0}}
$$

когда $z$ стремится к $z_{0}$, оставаясь в $\mathscr{F}$. Функция $f$ дифферени ируема на множестве $\mathscr{F}$, если она дифференцируема в любой его точке. Очевидно, что если $f$ дифференцируема на $\mathscr{F}$, то $f$ аналитична в области int $\mathscr{F}$. Обозначим через $C^{\infty}(\mathscr{F})$ множество всех функций, бесконечно дифференцируемых на $\mathscr{F}$.

Борелем [1] была поставлена следуюшая задача: по произвольной последовательности $\left\{d_{n}\right\}_{n=0}^{\infty}$ комплексных чисел найти функцию $y(z)$ из $C^{\infty}(\mathscr{F})$ такую, что

$$
y^{(n)}(0)=d_{n}, \quad n=0,1,2, \ldots .
$$

Если эта задача разрешима в $C^{\infty}(\mathscr{F})$ для любой последовательности $\left\{d_{n}\right\}_{n=0}^{\infty}$, то множество $\mathscr{F}$ будем называть $B$-множеством. Легко заметить, что если $\mathscr{F}-B$-множество, то точка $z=0$ должна быть граничной для $\mathscr{F}$. Действительно, допустим, что $0 \in \operatorname{int} \mathscr{F}$, и выберем последовательность $\left\{d_{n}\right\}_{n=0}^{\infty}$ такую, что

$$
\varlimsup_{n \rightarrow \infty}\left|\frac{d_{n}}{n !}\right|^{1 / n}=\infty
$$

Работа выполнена при финансовой поддержке Российского фонда фундаментальных исследований, гранты № 96-01-01041 и № 99-01-01018. 
Пусть $y_{0}(z)$ - решение из $C^{\infty}(\mathscr{F})$ задачи $(1)$. Тогда для всех $n \geqslant 0$ вьполняется равенство $d_{n}=y_{0}^{(n)}(0)$, откуда

$$
\varlimsup_{n \rightarrow \infty}\left|\frac{d_{n}}{n !}\right|^{1 / n}=\varlimsup_{n \rightarrow \infty}\left|\frac{y_{0}^{(n)}(0)}{n !}\right|^{1 / n} \leqslant \frac{1}{\rho(0, \partial \mathscr{F})}<\infty,
$$

где $\rho(0, \partial \mathscr{F})$ - расстояние от точки $z=0$ до границы $\partial \mathscr{F}$ множества $\mathscr{F}$, и мы пришли к противоречию. В дальнейшем рассматриваются плотные в себе множества с непустой связной внутренностью, граница которых содержит точку $z=0$.

В исходной работе [1] было показано, что для любой последовательности $\left\{d_{n}\right\}_{n=0}^{\infty}$ можно построить функцию $y(z)$, аналитическую в круге $|z-1|<1$, бесконечно дифференцируемую на множестве $|z-1| \leqslant 1$ и удовлетворяющую соотношениям (1). В последующем проблема Бореля исследовалась, как правило, в классе $E$, где $E$ - пространство функций, бесконечно дифференцируемых на некотором интервале вещественной оси или на всей оси, либо его подпространство (типа пространств Берлинга и Румье; см., например, [2], [3]). По пространству $E$ определялось естественным образом некоторое пространство $H$ числовых последовательностей такое, что для любой функции $f \in E$ последовательность $\Pi_{f}:=\left\{f^{(n)}(0)\right\}_{n=0}^{\infty} \in H$, и выяснялось, когда оператор П сюръективен или имеет линейньй непрерывный правый обратный.

Что же касается аналитических решений проблемы Бореля, их явного представления, характера расположения особых точек, то эти вопросы, насколько нам известно, не рассматривались.

То же относится и к многомерной проблеме Бореля, о которой пойдет речь ниже.

В настоящей работе решение задачи (1) строится в виде различных функциональных рядов, структура которых определяет соответствующее им $B$-множество. В качестве таких рядов используются последовательно степенные ряды, ряды по простейшим дробям и ряды экспонент. Исследуется характер аналитичности решения (наличие особых точек на гранище $\mathscr{F})$. При этом существование функции, представимой в виде соответствующего ряда и удовлетворяющей соотношениям (1), устанавливаются одним и тем же приемом, основанным на использовании следующей теоремы (см., например, [4, гл. $2, \S 2.5])$.

ТЕОРЕма ПолИА. Пусть дана бесконечная система линейных уравнений

$$
\sum_{j=1}^{\infty} a_{i j} u_{j}=b_{i}, \quad i=1,2, \ldots
$$

где $\left\{b_{j}\right\}_{j \geqslant 1}$ - произвольная последовательность, акоэффичиенты $\left\{a_{i j}\right\}$ удовлетворяют условиям:

I) первая строка $\left\{a_{1 j}\right\}_{j=1}^{\infty}$ содержит бесконечное число отличных от нуля әлементов;

II) $\varliminf_{j \rightarrow \infty} \frac{\left|a_{1 j}\right|+\left|a_{2 j}\right|+\cdots+\left|a_{i-1, j}\right|}{\left|a_{i j}\right|}=0, i=2,3, \ldots$

Тогда существует решение $\left\{u_{j}\right\}_{j=1}^{\infty}$ системы (3) такое, что все ряды в левых частях уравнений (3) абсолютно сходятся.

Решение задачи (1), как правило, будет отыскиваться в пространстве $C_{b}^{\infty}(\mathscr{F})$ функций из $C^{\infty}(\mathscr{F})$, ограниченных на $\mathscr{F}$ вместе с каждой своей производной. Топология в $C_{b}^{\infty}(\mathscr{F})$ задается счетным набором норм $\|y\|_{n}=\sup \left\{\left|y^{(k)}(z)\right|: z \in \mathscr{F}, k=0,1, \ldots, n\right\}$, $n=0,1,2, \ldots$, и в этой топологии $C_{b}^{\infty}(\mathscr{F})$ является пространством $\Phi$ реше. 
2. Пусть $\alpha \in \mathbb{C}, \alpha \neq 0, K_{\alpha}:=\{z:|z-\alpha|<|\alpha|\}, \bar{K}_{\alpha}=\{z:|z-\alpha| \leqslant|\alpha|\}$. Пусть, далее, для всех $n \geqslant 1 m_{n}$ - натуральные числа такие, что $0 \leqslant m_{1}<m_{2}<\cdots$. Решение задачи $(1)$ в классе $C^{\infty}\left(\bar{K}_{\alpha}\right)$ будем искать в виде

$$
y(z)=\sum_{n=1}^{\infty} c_{n}(z-\alpha)^{m_{n}}
$$

Рассмотрим вспомогательную систему

$$
\sum_{\substack{n=1 \\ m_{n} \geqslant k}}^{\infty} \frac{c_{n} m_{n} !}{\left(m_{n}-k\right) !}(-1)^{m_{n}-k} \alpha^{m_{n}-k}=d_{k}, \quad k=0,1,2, \ldots
$$

Фиксируем $s \geqslant 2$ и $\varepsilon>0$. Пусть сначала $n$ таково, что $m_{n}>2 s$. Положим

$$
\sigma_{s, n}=\sum_{k=0}^{s-1} \frac{m_{n} !}{\left(m_{n}-k\right) !}|\alpha|^{m_{n}-k}, \quad \gamma_{s, n}=\frac{m_{n} !}{\left(m_{n}-s\right) !}|\alpha|^{m_{n}-s}, \quad \tau_{s, n}=\frac{\sigma_{s, n}}{\gamma_{s, n}} .
$$

Нетрудно подсчитать, что

$$
\begin{array}{ll}
\tau_{s, n}<\frac{|\alpha|\left(|\alpha|^{s}-1\right)}{\left(m_{n}-s+1\right)(|\alpha|-1)} & \text { при }|\alpha| \neq 1, \\
\tau_{s, n}<\frac{s}{m_{n}-s+1} & \text { при }|\alpha|=1 .
\end{array}
$$

Выберем номер $N$ так, чтобы $\varepsilon\left(m_{N}-s+1\right)>\tau_{s}$, где

$$
\tau_{s}= \begin{cases}s & \text { при }|\alpha|=1 \\ \frac{|\alpha|\left(|\alpha|^{s}-1\right)}{|\alpha|-1} & \text { при }|\alpha| \neq 1\end{cases}
$$

Тогда для всех $n \geqslant N$ выполнено неравенство $\tau_{s, n}<\varepsilon$, откуда $\varlimsup_{n \rightarrow \infty} \tau_{s, n} \leqslant \varepsilon$. Устремляя $\varepsilon>0$ к нулю, находим, что условие II) в теореме Полиа вьполнено. Очевидно, что условие I) для системы (5) также выполнено, и потому она имеет бесконечное множество решений $\left\{c_{n}\right\}_{n=1}^{\infty}$ таких, что

$$
\sum_{\substack{n=1 \\ m_{n} \geqslant k}}^{\infty} \frac{\left|c_{n}\right| m_{n} !}{\left(m_{n}-k\right) !}|\alpha|^{m_{n}-k}<\infty, \quad k=0,1,2, \ldots
$$

Пусть $\left\{c_{n}\right\}_{n=1}^{\infty}-$ одно из этих решений. Образуем по нему ряд (4). Очевидно, что он сходится абсолютно в $C_{b}^{\infty}\left(\bar{K}_{\alpha}\right)$. Следовательно, $y(z) \in C_{b}^{\infty}\left(\bar{K}_{\alpha}\right)$, и $y(z)$-искомое решение задачи (1). Сформулируем полученный результат.

ТЕорема 1. При любом $\alpha \neq 0 \bar{K}_{\alpha}-$ В-множество. Для произвольной последовательности $\left\{d_{k}\right\}_{k=0}^{\infty}$ комплексных чисел решение проблемы Бореля (1) из $C_{b}^{\infty}\left(\bar{K}_{\alpha}\right)$ представляется в виде ряда (4). 
ЗАмечАнИЕ. То, что $K_{1}$ является $B$-множеством, фактически доказано самим Борелем [1] другим способом, но также с помощью степенных рядов.

Назовем начальные данные $\left\{d_{n}\right\}_{n=0}^{\infty}$ неаналитическими, если вьполнено условие (2), и аналитическими в противном случае.

Пусть начальные данные $\left\{d_{n}\right\}_{n=0}^{\infty}$ неаналитические, $\alpha \neq 0$ и $y(z)$ - произвольное решение задачи $(1)$ из $C^{\infty}\left(\bar{K}_{\alpha}\right)$. Тогда $y(z)$ аналитична в круге $K_{\alpha}$. Покажем, что точка $z=0$ особая для $y(z)$. Рассуждая от противного, допустим, что $z=0$ - правильная точка $y(z)$. Тогда найдется функция $y_{1}(z)$, аналитическая в круге $E_{\rho}:=\{z:|z|<\rho\}$, $\rho>0$, и совпадающая с $y(z)$ в $D_{\rho}:=K_{\alpha} \cap E_{\rho}$. Но тогда для всех $k \geqslant 0$ вьполнено

$$
d_{k}=\lim _{\substack{z \rightarrow 0 \\ z \in \bar{K}_{\alpha}}} y^{(k)}(z)=\lim _{\substack{z \rightarrow 0 \\ z \in D \rho}} y^{(k)}(z)=\lim _{\substack{z \rightarrow 0 \\ z \in D_{\rho}}} y_{1}^{(k)}(z)=\lim _{z \rightarrow 0} y_{1}^{(k)}(z)=y_{1}^{(k)}(0) .
$$

Отсюда

$$
\varlimsup_{k \rightarrow \infty}\left|\frac{d_{k}}{k !}\right|^{1 / k}=\varlimsup_{k \rightarrow \infty}\left(\frac{\left|y_{1}^{(k)}(0)\right|}{k !}\right)^{1 / k} \leqslant \frac{1}{\rho},
$$

и мы пришли к противоречию.

Этот простой результат можно дополнить.

Теорема 2. Пусть $\alpha \neq 0$ и начальные данные $\left\{d_{n}\right\}_{n=0}^{\infty}$ неаналитические. Тогда найдется решение $y(z)$ задачи (1) из $C_{b}^{\infty}\left(\bar{K}_{\alpha}\right)$ в виде ряда (4) такое, что все точки окружности $|z-\alpha|=|\alpha|$ являются особыми для $y(z)$.

ДокАЗАТЕльство. Выберем последовательность натуральных чисел $\left\{m_{n}\right\}_{n=1}^{\infty}$ так, чтобы $\lim _{n \rightarrow \infty}\left(n / m_{n}\right)=0$. Пусть $y(z)-$ сумма ряда $(4)$, в котором $\left\{c_{n}\right\}_{n=1}^{\infty}-$ одно из решений системы (5) такое, что для него выполнено условие (6). По известной теореме Фату о лакунах (см., например, [5, с. 80-81]) область существования функции $y(z)$ совпадает с (открытьм) кругом сходимости $|z-\alpha|<R$ ряда (4). Очевидно, что $R \geqslant|\alpha|$. Допустим, что $R>\alpha$. Тогда $y(z)$ аналитична в круге $|z-\alpha|<R$, содержащем точку $z=0$, и поэтому при всех $k \geqslant 0 d_{k}=y^{(k)}(0)$. Отсюда

$$
\varlimsup_{k \rightarrow \infty}\left|\frac{d_{k}}{k !}\right|^{1 / k}=\varlimsup_{k \rightarrow \infty}\left|\frac{y^{(k)}(0)}{k !}\right|^{1 / k} \leqslant \frac{1}{R-|\alpha|}<\infty,
$$

и мы пришли к противоречию. Следовательно, $R=|\alpha|$, и окружность $|z-\alpha|=|\alpha|$ является естественной границей (купюрой) вейерштрассовой области существования $y(z)$.

СледСТвИЕ. Пусть $0<R<\infty, \alpha \in \mathbb{C} u K_{R}(\alpha)=\{z:|z-\alpha|<R\}, \overline{K_{R}(\alpha)}=\{z:$ $|z-\alpha| \leqslant R\}$. Тогда для любой граничной точки $\beta$ круга $K_{R}(\alpha)$ и для любых неаналитических начальных данных $\left\{d_{n}\right\}_{n=0}^{\infty}$ найдется ряд $\sum_{n=0}^{\infty} c_{n}(z-\alpha)^{n}$ такой, что радиус его сходимости равен $R$, каждая точка окружности $|z-\alpha|=R$ - особая для суммы $v(z)$ этого ряда, $v(z) \in C_{b}^{\infty}\left(\overline{K_{R}(\alpha)}\right) u v^{(n)}(\beta)=d_{n}, n=0,1,2, \ldots$

3. Пусть теперь $G$ - односвязная область с замкнутой жордановой гранищей $\Gamma$ класса $C^{\infty}$, содержащей точку $z=0$. Пусть, далее, функция $\varphi(z)$ конформно отображает $G$ на круг $K_{1}$ (и, следовательно, отображает взаимно однозначно и взаимно непрерьвно $\bar{G}$ на $\left.\overline{K_{1}}\right)$ так, что $\varphi(0)=0$. Как известно, $\varphi \in C_{b}^{\infty}(\bar{G})$. Если $f \in C_{b}^{\infty}\left(\overline{K_{1}}\right)$ и $g(z):=f(\varphi(z))$, то $g(0)=f(0)$ и при всех $k \geqslant 1$ вьполняется соотношение $g^{(k)}(0)=$ $\sum_{s=1}^{k} \alpha_{s, k} f^{(s)}(0)$, где $\alpha_{k, k}=\left(\varphi^{\prime}(0)\right)^{k}$ и $\alpha_{1, k}=\varphi^{(k)}(0)$. Отсюда $f(0)=g(0)$ и для всех 
$k \geqslant 1$ вьполняется соотношение $f^{(k)}(0)=\sum_{s=1}^{k} \beta_{s, k} g^{(k)}(0)$, где $\beta_{k, k} \neq 0$. Зададим произвольно последовательность $\left\{d_{k}\right\}_{k=0}^{\infty}, d_{k} \in \mathbb{C}$, и положим $\widetilde{d}_{0}=d_{0}, \widetilde{d}_{k}=\sum_{s=1}^{k} \beta_{s, k} d_{s}$, $k \geqslant 1$. В силу теоремы 1 существует функция $y(z)$ из $C_{b}^{\infty}\left(\overline{K_{1}}\right)$ вида $(4)$ (с $\left.\alpha=1\right)$ такая, что $y^{(k)}(0)=\widetilde{d}_{k}, k=0,1,2, \ldots$ Тогда $g=f \circ \varphi \in C_{b}^{\infty}(\bar{G})$ и $g^{(k)}(0)=d_{k}, k=0,1,2, \ldots$. Таким образом, $g$ - решение задачи (1) из $C_{b}^{\infty}(\bar{G})$ и $\bar{G}-B$-множество.

Наиболее простьм и, пожалуй, наиболее важньм примером $B$-множества, полученного таким путем, может служить множество $|z-\alpha| \geqslant|\alpha|, \alpha \in \mathbb{C}, \alpha \neq 0$, а также любая замкнутая полуплоскость, граница которой содержит начало координат. В качестве функции $\varphi(z)$ здесь берется соответствующая дробно-линейная функция $\alpha z /(\gamma z+\delta)$, a само решение задачи (1) имеет вид

$$
\sum_{n=1}^{\infty} c_{n}\left(\frac{\alpha z}{\gamma z+\delta}\right)^{n}
$$

Например, если $G=\{z:|z-\alpha|>|\alpha|\}$, то $\varphi(z)=z /(z-\alpha)$, а если $G=\{z: \operatorname{Re} z<0\}$, то $\varphi(z)=2 z /(z-1)$.

4. Исследуем теперь решения задачи (1), используя ряды по простейшим дробям.

Пусть $\alpha_{k} \neq \alpha_{m}$ при $k \neq m, \alpha_{k} \in \mathbb{C}, k=1,2, \ldots, \lim _{k \rightarrow \infty} \alpha_{k}=0$. Положим

$$
v(z)=\sum_{k=1}^{\infty} \frac{c_{k}}{z-\alpha_{k}}
$$

и рассмотрим соответствующую вспомогательную систему

$$
-\sum_{k=1}^{\infty} j ! c_{k} \alpha_{k}^{-j-1}=d_{j}, \quad j=0,1,2, \ldots
$$

Чтобы применить теорему Полиа, зафиксируем $s \geqslant 2$. Тогда

$$
\delta_{k, s}:=\sum_{j=0}^{s-1} j !\left|\alpha_{k}\right|^{-j-1} \leqslant(s-1) !\left|\alpha_{k}\right|^{-s} d, \quad \text { где } \quad d=\exp \max _{k \geqslant 1}\left|\alpha_{k}\right| .
$$

Отсюда

$$
\frac{\delta_{k, s}\left|\alpha_{k}\right|^{s+1}}{s !} \leqslant \frac{d\left|\alpha_{k}\right|}{s} \leqslant \frac{d\left|\alpha_{k}\right|}{2}<\varepsilon \quad \forall k>N(\varepsilon),
$$

и условие II) теоремы Полиа выполнено. Согласно последней система (8) имеет бесконечное множество решений $\left\{c_{k}\right\}_{k=1}^{\infty}$ таких, что

$$
\sum_{k=1}^{\infty}\left|c_{k}\right| \cdot\left|\alpha_{k}\right|^{-j-1}<\infty, \quad j=0,1,2, \ldots
$$

Зафиксируем любое число $\eta$ из $(0,1)$ и положим

$$
B_{k}^{\eta}=\left\{z \in \mathbb{C}:\left|z-\alpha_{k}\right| \geqslant \eta\left|\alpha_{k}\right|\right\}, \quad B_{n}(\eta)=\bigcap_{k=n}^{\infty} B_{k}^{\eta}, \quad n=1,2, \ldots
$$


Если $v(z)$ - сумма ряда $(7)$, в котором $\left\{c_{k}\right\}_{k=1}^{\infty}-$ какое-либорешение системы (8) со свойством (9), то ряд (7) сходится абсолютно в $C_{b}^{\infty}\left(B_{1}(\eta)\right), \eta \in(0,1)$. При этом $0 \in \partial B_{1}(\eta)$ (и вообще $0 \in \partial B_{n}(\eta)$ для всех $n \geqslant 1$ ). Функцию $v(z)$, являющуюся решением задачи $(1)$ из $C_{b}^{\infty}\left(B_{1}(\eta)\right)$, можно представить в виде

$$
v(z)=\sum_{k=1}^{N} \frac{c_{k}}{z-\alpha_{k}}+\sum_{k=N+1}^{\infty} \frac{c_{k}}{z-\alpha_{k}}=v_{N}(z)+w_{N}(z), \quad N=1,2, \ldots
$$

Учитьвая (9), находим, что ряд

$$
\sum_{k=N+1}^{\infty} \frac{c_{k}}{z-\alpha_{k}}
$$

сходится абсолютно в $C_{b}^{\infty}\left(B_{N+1}(\eta)\right)$, и потому $w_{N} \in C_{b}^{\infty}\left(B_{N+1}(\eta)\right)$. Что же касается функции $v_{N}$, то она аналитична в расширенной комплексной плоскости, из которой удалено множество $\Lambda_{N}:=\left\{\alpha_{k}: k=1,2, \ldots, N\right\}$. Положим $\Lambda=\{0\} \cup\left\{\alpha_{k}: k=1,2, \ldots\right\}$. Неограниченно увеличивая $N$ и уменьшая $\eta>0$, получаем, что $v \in \mathscr{A}(\overline{\mathbb{C}} \backslash \Lambda)$, где для любой области $G$ в $\overline{\mathbb{C}} \mathscr{A}(G)$ - пространство функций, аналитических в $G$. При этом для всех $N \geqslant 1$ выполняется равенство $v(z)=v_{N}(z)+w_{N}(z)$, где $v_{N} \in \mathscr{A}\left(\overline{\mathbb{C}} \backslash \Lambda_{N}\right)$, $w_{N} \in C_{b}^{\infty}\left(B_{N+1}(\eta)\right)$ при любом $\eta>0$. Далее, для всех $k \geqslant 0$ вьполняется равенство $v^{(k)}(0)=d_{k}=\lim _{z \rightarrow 0} v^{(k)}(z)$, когда $z$ стремится к началу координат, оставаясь на множестве $B_{N+1}(\eta), N \geqslant 1$.

Положим $\Phi=\left\{1 /\left(z-\alpha_{k}\right): k=1,2, \ldots\right\}$, и пусть $\operatorname{span} \Phi-$ совокупность всех конечных линейных комбинаций функций из $\Phi$. Легко заметить, что $z=0$-правильная точка для суммы $v(z)$ ряда (7) тогда и только тогда, когда ряд вырождается в конечную сумму, т.е. когда для всех $j \geqslant 0$ выполнено равенство $d_{j}=g^{(j)}(0)$, где $g(z) \in \operatorname{span} \Phi$. Если начальные данные $\left\{d_{j}\right\}_{j=0}^{\infty}$ не имеют такой структуры (в частности, если они неаналитические), то точка $z=0$ особая для любого решения задачи (1) вида (7) (так как $z=0$-предельная точка множества полюсов $v(z))$.

Заметим, что при любом фиксированном $N \geqslant 1$ связность множества $B_{N+1}(\eta)$ может быть сделана как угодно большой за счет уменьшения $\eta$.

Используя рассуждения данного пункта, нетрудно указать условия довольно общего характера, при выполнении которых $\mathscr{F}$ будет $B$-множеством.

Теорема 3. Пусть $\mathscr{F}$ - плотное в себе множество в $\mathbb{C} u z=0$ - граничная

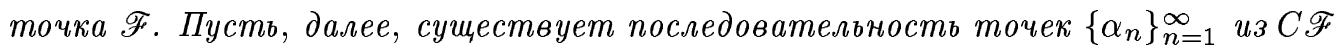
maкая, чmo

$$
\lim _{n \rightarrow \infty} z_{n}=0, \quad a:=\sup _{n \geqslant 1} \frac{\left|\alpha_{n}\right|}{\rho\left(\alpha_{n}, \mathscr{F}\right)}<\infty .
$$

Тогда $\mathscr{F}-$ В-мнохество.

ДокАЗАТЕльство. Зададим какую-либо последовательность $\left\{d_{n}\right\}_{n=0}^{\infty}$ и образуем ряд (7), коэффициенты $c_{k}$ которого удовлетворяют системе уравнений (8) и условиям (9). Пусть $\eta_{0} \in(0,1 / 2 a) \cap(0,1)$. Тогда для всех $z \in \mathscr{F}, n \geqslant 1$ выполнена оценка $\left|z-\alpha_{n}\right| \geqslant \rho\left(\alpha_{n}, \mathscr{F}\right)>\left|\alpha_{n}\right| \eta_{0}$. Следовательно, $\mathscr{F} \subset B_{1}\left(\eta_{0}\right)$, откуда $v(z) \in C_{b}^{\infty}(\mathscr{F})$, причем для любого $k \geqslant 0$

$$
v^{(k)}(0)=d_{k}=\lim _{z \rightarrow 0} v^{(k)}(z)
$$


когда $z$ стремится к началу координат, оставаясь на множестве $\mathscr{F}$.

При этом $v(z)$ как сумма ряда (7) и в силу условий (9) определена как однозначная и локально аналитическая функция на множестве $\mathbb{C} \backslash \Lambda$ (и подавно на $\mathscr{F} \backslash\{0\})$ : для каждого $z_{0}$ из $\mathbb{C} \backslash \Lambda$ функция $v(z)$ аналитична в круге $\left|z-z_{0}\right|<\rho\left(z_{0}, \Lambda\right)$.

5. Обратимся, наконец, к представлению решения задачи (1) в виде ряда экспонент. Зафиксируем какую-либо последовательность $\left\{\lambda_{k}\right\}_{k=1}^{\infty}$ такую, что

$$
\lambda_{k} \in \mathbb{R} \quad \forall k \geqslant 1 ; \quad \lambda_{k} \neq \lambda_{n} \quad \text { при } k \neq n ; \quad \lim _{k \rightarrow \infty}\left|\lambda_{k}\right|=\infty .
$$

Решение задачи (1) ищется теперь в виде ряда

$$
v(z)=\sum_{k=1}^{\infty} c_{k} e^{i \lambda_{k} z}
$$

в котором $\left\{c_{k}\right\}_{k=1}^{\infty}-$ любая последовательность комплексных чисел, удовлетворяющая системе

$$
\sum_{k=1}^{\infty} c_{k}\left(i \lambda_{k}\right)^{n}=d_{n}, \quad n=0,1,2, \ldots
$$

и такая, что

$$
\sum_{k=1}^{\infty}\left|c_{k}\right| \cdot\left|\lambda_{k}\right|^{n}<\infty, \quad n=0,1,2, \ldots
$$

Подобная последовательность всегда найдется в силу теоремы Полиа (проверка вьполнения ее условий I),II) для системы (12) не вызывает никаких затруднений). Ясно, что ряд (11) сходится абсолютно в пространстве $C_{b}^{\infty}(\mathbb{R})$. Очевидно также, что $v \in C_{b}^{\infty}(\mathbb{R})$.

Опишем структуру решения $v$ задачи Бореля (1). Пусть $\mathbb{N}_{1}=\left\{k \geqslant 1: \lambda_{k}>0\right\}$, $\mathbb{N}_{2}=\left\{k \geqslant 1: \lambda_{k} \leqslant 0\right\}$. Тогда

$$
v(z)=v_{1}(z)+v_{2}(z)=\sum_{k \in \mathbb{N}_{1}} c_{k} e^{i \lambda_{k} z}+\sum_{k \in \mathbb{N}_{2}} c_{k} e^{i \lambda_{k} z} .
$$

Ясно, что $v_{j}(z) \in C_{b}^{\infty}(\mathbb{R}), j=1,2$, и оба ряда $(13)$ сходятся абсолютно в $C_{b}^{\infty}(\mathbb{R})$. При этом первый из них сходится абсолютно в $C_{b}^{\infty}\left(\bar{\Pi}_{+}\right)$, а второй - в $C_{b}^{\infty}\left(\bar{\Pi}_{-}\right)$, где $\bar{\Pi}_{+}=\{z: \operatorname{Im} z \geqslant 0\}, \bar{\Pi}_{-}=\{z: \operatorname{Im} z \leqslant 0\}$.

Таким образом, любое решение $v$ задачи Бореля (1) вида (11) имеет представление $v(z)=v_{1}(z)+v_{2}(z)$, где $v_{1}(z) \in C_{b}^{\infty}\left(\bar{\Pi}_{+}\right), v_{2}(z) \in C_{b}^{\infty}\left(\bar{\Pi}_{-}\right)$. Если одно из множеств $\mathbb{N}_{1}, \mathbb{N}_{2}$ пусто, то соответствующая функция $v_{j}(z)$ в этом представлении отсутствует.

Построим теперь решение задачи (1) также в виде ряда экспонент, но с несколько иными свойствами. Возьмем последовательность $\left\{\lambda_{k}\right\}_{k=1}^{\infty}$ такую, что

$$
\lambda_{k} \geqslant 0 \quad \forall k \geqslant 1 ; \quad \lambda_{k} \neq \lambda_{n} \quad \text { при } k \neq n ; \quad \lim _{k \rightarrow \infty} \lambda_{k}=\infty .
$$

Тогда сумма $w(z)$ ряда

$$
w(z)=\sum_{k=1}^{\infty} c_{k} e^{\lambda_{k} z}
$$


в котором $\left\{c_{k}\right\}_{k=1}^{\infty}-$ какое-либо решение системы

$$
\sum_{k=1}^{\infty} c_{k}\left(\lambda_{k}\right)^{n}=d_{n}, \quad n=0,1,2, \ldots
$$

такое, что

$$
\sum_{k=1}^{\infty}\left|c_{k}\right|\left(\lambda_{k}\right)^{n}<\infty, \quad n=0,1,2, \ldots
$$

принадлежит пространству $C_{b}^{\infty}\left(\bar{\Pi}_{l}\right)$, где $\bar{\Pi}_{l}=\{z: \operatorname{Re} z \leqslant 0\}$, и является решением задачи $(1)$, причем ряд в правой части $(15)$ сходится абсолютно в $C_{b}^{\infty}\left(\bar{\Pi}_{l}\right)$. Пусть еще $w_{1}(z)=\sum_{k=1}^{\infty} c_{k, 1} e^{\lambda_{k} z}-$ полученное тем же способом решение из $C_{b}^{\infty}\left(\bar{\Pi}_{l}\right)$ задачи $(1)$, но с начальными данными $(-1)^{n} d_{n}, n=0,1,2, \ldots$ Тогда функция

$$
w_{2}(z):=w_{1}(-z)=\sum_{k=1}^{\infty} c_{k, 1} e^{-\lambda_{k} z}
$$

принадлежит пространству $C_{b}^{\infty}\left(\bar{\Pi}_{r}\right)$, где $\bar{\Pi}_{r}=\{z: \operatorname{Re} z \geqslant 0\}$, причем при всех $n \geqslant 0$ выполнено равенство $w_{2}^{(n)}(0)=(-1)^{n} w_{1}^{(n)}(0)=d_{n}$.

Назовем функцию $f(x)=w(x+0 \cdot i)$, определенную на $(-\infty, 0]$, следом функции $w$ на полуоси $(-\infty, 0]$. Аналогично определяется след $f_{2}(x)=w_{2}(x+0 \cdot i)$ функции $w_{2}$ на положительной полуоси. Функция $f_{3}(x)$, равная $f(x)$ на $(-\infty, 0]$ и $f_{2}(x)-$ на $(0,+\infty)$, принадлежит $C_{b}^{\infty}(\mathbb{R})$ и является решением задачи (1). Оценим порядок ее убывания на бесконечности. Если $x<0$, то

$$
\left|f_{3}(x)\right|=|f(x)| \leqslant\left(\sum_{k=1}^{\infty}\left|c_{k}\right|\right) \cdot e^{\lambda_{1} x}
$$

так как числа $\lambda_{k}>0$ всегда можно перенумеровать в порядке их возрастания. При этом последовательность $\left\{\lambda_{n}\right\}_{n=1}^{\infty}$ можно выбрать так, чтобы $N \leqslant \lambda_{1}<\lambda_{2}<\cdots$, где $N$ - любое фиксированное число, которое можно взять сколь угодно большим. Следовательно, $\varlimsup_{x \rightarrow-\infty}\left|f_{3}(x)\right| e^{-N x}<\infty$. Аналогично, если $x>0$, то

$$
\left|f_{3}(x)\right|=\left|f_{2}(x)\right| \leqslant\left(\sum_{k=1}^{\infty}\left|c_{k, 1}\right|\right) \cdot e^{-\lambda_{1} x} \leqslant A e^{-N x}
$$

и, окончательно, $\varlimsup_{|x| \rightarrow \infty}\left|f_{3}(x)\right| e^{N|x|}<\infty$.

Назовем число

$$
\rho_{f_{3}}:=\varlimsup_{|x| \rightarrow \infty} \frac{\ln \left|f_{3}(x)\right|}{|x|}
$$

степенью әкспоненииального убывания на бесконечности функции $f_{3}$.

Подведем итог рассуждениям, проведенным до сих пор в этом пункте. 
ТЕоремА 4. Для любой последовательности $\left\{d_{n}\right\}_{n=0}^{\infty}$ комплексных чисел найдутся решения из $C_{b}^{\infty}(\mathbb{R})$ задачи (1) следуюших типов:

1) решение $v$ в виде абсолютно сходящегося в $C_{b}^{\infty}(\mathbb{R})$ ряда $(10),(11)$, представимое в виде $v(z)=v_{1}(z)+v_{2}(z)$, где $v_{1}(z) \in C_{b}^{\infty}\left(\bar{\Pi}_{+}\right), v_{2}(z) \in C_{b}^{\infty}\left(\bar{\Pi}_{-}\right)$;

2) решение $f_{3}(x)$, которое на $(-\infty, 0]$ совпадает со следом функиии $w(z)$ из $C_{b}^{\infty}\left(\bar{\Pi}_{l}\right)$, а на $(0,+\infty)$ - со следом функиии $w_{2}(z)$ из $C_{b}^{\infty}\left(\bar{\Pi}_{r}\right) ;$ при этом существуют решения $f_{3}$ такого типа со сколь угодно большой степенью экспоненциального убывания на бесконечности.

Продолжим исследование свойств решений задачи $(1)$ из $C_{b}^{\infty}\left(\bar{\Pi}_{l}\right)$ в виде рядов (14), (15). Как и в п. 2 (перед формулировкой теоремы 2), получаем, что если начальные данные $\left\{d_{n}\right\}_{n=0}^{\infty}$ неаналитические и если $y(z)$ - любое решение задачи (1) из $C^{\infty}\left(\bar{\Pi}_{l}\right)$, то $z=0$ - особая точка $y(z)$. Как и вьше, этот результат можно дополнить.

Теорема 5. Пусть показатели $\lambda_{n}$ таковы, что

$$
\lambda_{n} \neq \lambda_{m} \quad \text { npu } n \neq m ; \quad \lambda_{n} \geqslant 0, \quad n=1,2, \ldots ; \quad \lim _{n \rightarrow \infty} \frac{n}{\lambda_{n}}=0 .
$$

Пусть далее, $\left\{d_{n}\right\}_{n=0}^{\infty}-$ произвольная последовательность комплексньх чисел, $a$ $v(z)$ - какое-либо решение задачи (1) в виде ряда (15) с коэффиииентами $\left\{c_{k}\right\}_{k=1}^{\infty}$, удовлетворяющими системе (16) и такими, что $\sum_{k=1}^{\infty}\left|c_{k}\right|<\infty$. Тогда или v(z)иелая функиия, или ее вейерштрассова область существования - некоторая полуплоскость $\operatorname{Re} z<d, d \geqslant 0$. При әтом если начальные данные неаналитические, то вейерштрассова область существования $v(z)$ совпадает с полуплоскосmью $\operatorname{Re} z<0$.

ДоКАЗАТЕЛЬСТВо. Из предположений теоремы следует, что ряд (15) сходится абсолютно на множестве $\bar{\Pi}_{l}$. По одной из теорем все того же Полиа [6] в этом случае в силу условий (17) или $v(z)$ - целая функция, или вейерштрассова область существования функции $v(z)$ совпадает с некоторой полуплоскостью $\Pi_{d}:=\{z: \operatorname{Re} z<d\}$. Очевидно, что $d \geqslant 0$.

Пусть теперь $\left\{d_{n}\right\}_{n=0}^{\infty}-$ неаналитические начальные данные. Допустим, что $v(z)$ аналитична в открытой полуплоскости, содержащей $\bar{\Pi}_{0}$ (т.е. или $v(z)$ - целая функция, или ее область существования - полуплоскость $\left.\Pi_{d} \mathrm{c} d>0\right)$. Тогда $v(z)$ аналитически продолжается из $\Pi_{0}$ в круг $|z|<d$, откуда

$$
\varlimsup_{n \rightarrow \infty}\left|\frac{d_{n}}{n !}\right|^{1 / n}=\varlimsup_{n \rightarrow \infty}\left|\frac{v^{(n)}(0)}{n !}\right|^{1 / n} \leqslant \frac{1}{d}<\infty,
$$

что невозможно. Следовательно, $d=0$ и все точки мнимой оси особые для $v(z)$.

Зафиксируем какое-либо $\varphi$ из $[0,2 \pi)$ и рассмотрим последовательность $\mu_{k}(\varphi)=\lambda_{k} e^{i \varphi}$, где числа $\left\{\lambda_{k}\right\}_{k=1}^{\infty}$ удовлетворяют условиям (14) или (17). Тогда решение задачи (1) ищется в виде ряда (15), в котором показатели $\lambda_{k}$ заменены на $\mu_{k}(\varphi)$. Ясно, что замена $z e^{i \varphi}=w$ приводит этот случай к ранее рассмотренному. Таким образом, для любого $\varphi$ из $[0,2 \pi)$ и для любых начальньх данных $\left\{d_{n}\right\}_{n=0}^{\infty}$ существует решение задачи $(1)$ из $C_{b}^{\infty}\left(\bar{\Pi}^{\varphi}\right)$, где $\Pi^{\varphi}=\left\{z: \operatorname{Re} z e^{i \varphi}<0\right\}, \bar{\Pi}^{\varphi}=\left\{z: \operatorname{Re} z e^{i \varphi} \leqslant 0\right\}$. Таким путем можно получить аналог теоремы 4 , в котором вещественная прямая $\mathbb{R}$ заменяется прямой $\operatorname{Im} z e^{i \varphi}=0$. Справедлив и аналог теоремы 5. 
6. Пусть $v(z)$ - решение задачи (1) в виде ряда (15) с экспонентами $\lambda_{n}$, удовлетворяюшими условиям (17), и коэффициентами $\left\{c_{k}\right\}_{k=1}^{\infty}$, выбранными, как в теореме 5. Положим

$$
a(z)=\prod_{k=1}^{\infty}\left(1-\frac{z^{2}}{\lambda_{k}^{2}}\right) .
$$

Согласно хорошо известньм фактам теории целых функций $a(z)$ - целая функция из класса $[1,0]$ всех целых функций минимального типа при порядке 1 . Положим

$$
(a(D) y)(z)=\sum_{k=0}^{\infty} a_{k} y^{(k)}(z), \quad \text { где } \sum_{k=0}^{\infty} a_{k} z^{k}=a(z) .
$$

Как известно [7], если $G$-произвольная область и $\mathscr{A}(G)$ - пространство Фреше всех аналитических в области $G$ функций с топологией равномерной сходимости на компактах $G$, то $a(D)$ - линейньй непрерывный оператор в $\mathscr{A}(G)$. Ясно, что любая конечная линейная комбинация экспонент $\left\{e^{ \pm \lambda_{k} z}, k=1,2, \ldots\right\}$ удовлетворяет уравнению $(a(D) y)(z)=0$ в $\mathbb{C}$. Если $v(z)$ - решение задачи (1) из теоремы 4 и

$$
v_{n}(z):=\sum_{k=1}^{n} c_{k} e^{-\lambda_{k} z}, \quad n=1,2, \ldots,
$$

то внутри $\Pi_{l}=\{z: \operatorname{Re} z<0\}$ равномерно $0=\left(a(D) v_{n}\right)(z) \rightarrow(a(D) v)(z)$. Поэтому решение $v$ задачи (1) из теоремы 4 удовлетворяет всюду в $\Pi_{l}$ линейному дифференциальному уравнению бесконечного порядка:

$$
\sum_{k=0}^{\infty} a_{k} v^{(k)}(z)=0, \quad z \in \Pi_{l}
$$

и является для него решением задачи Коши из $\mathscr{A}\left(\Pi_{l}\right)$, причем начальные данные задаются в граничной точке $\Pi_{l}$.

В связи с этим дополнением к теореме 4 уместно напомнить некоторые результаты из [8, с. 413-415]. Пусть $a(z)$-произвольная трансцендентная функция из класса $[1,0]$ с нулями $\left\{\lambda_{n}\right\}_{n=1}^{\infty}$ (не обязательно простыми). Пусть, далее, $\alpha$ - какой-либо частичньй предел последовательности $\left\{\arg \lambda_{k}\right\}_{k=1}^{\infty}$. Для любого $\delta$ из $[0, \pi / 2)$ определим полуплоскость $\Gamma^{\delta}:=\{z: \pi / 2-\delta<\arg z<3 \pi / 2-\delta\}$. Метод, используемый при доказательстве теоремы 9 в [8] (и, кстати, также основанньй на теореме Полиа из п. 1), позволяет фактически доказать, что для произвольной последовательности $\left\{c_{k}\right\}_{k=0}^{\infty}$ найдется бесконечное множество функций $y(z)$ со следующими свойствами:

1) $y \in \mathscr{A}\left(\Gamma^{\alpha}\right)$;

2) в любой точке открытой полуплоскости $\Gamma^{\alpha}$ функция $y(z)$ удовлетворяет уравнению

$$
\sum_{k=0}^{\infty} \frac{a^{(k)}(0) y^{(k)}(z)}{k !}=0 ;
$$

3) для любого $\delta$ из $(0, \pi / 2) y(z) \in C_{b}^{\infty}\left(\bar{\Gamma}_{\delta}^{\alpha}\right)$, где $\bar{\Gamma}_{\delta}^{\alpha}$ - замкнутьй сектор, содержаший $z=0$, задаваемый соотношением $\pi / 2-\alpha+\delta \leqslant \arg z \leqslant 3 \pi / 2-\alpha-\delta$;

4) для всех $\delta>0, k \geqslant 0$ вьполнено равенство

$$
\lim _{\substack{z \rightarrow 0 \\ z \in \Gamma_{\delta}^{\alpha}}} y^{(k)}(z)=y^{(k)}(0)=c_{k}
$$


Если же найдется бесконечное множество нулей $a(z)$ с одинаковым аргументом $\varphi$, то тем же методом можно построить (неединственную) функцию $y(z)$ со свойствами 1$), 2$ ) и такую, что $y \in C_{b}^{\infty}\left(\bar{\Gamma}^{\alpha}\right) ;$ для всех $k \geqslant 0$

$$
\lim _{\substack{z \rightarrow 0 \\ z \in \bar{\Gamma}^{\alpha}}} y^{(k)}(z)=y^{(k)}(0)=c_{k}
$$

где $\bar{\Gamma}^{\alpha}=\{z: \pi / 2-\alpha \leqslant \arg z \leqslant 3 \pi / 2-\alpha\}-$ замкнутая полуплоскость. Эти результаты показывают, что если уравнение $\sum_{k=0}^{\infty} a_{k} y^{(k)}(z)=0$ с символом $\sum_{k=0}^{\infty} a_{k} z^{k}$ из класса $[1,0]$ не вырождается в уравнение конечного порядка, то задание начальных условий (1) в граничной точке области аналитичности решения этого уравнения не определяет однозначно решение. Таким образом, известная в теории обыкновенньх дифференциальных уравнений теорема Брио и Буке (дополненная Пикаром и Пенлеве) для уравнения бесконечного порядка неверна.

7. Как известно, проблема Бореля была исследована и в многомерной ситуации в пространстве $C_{(\mathbb{R})}^{\infty}(Q)$ гладких вещественнозначных функций от $n$ вещественных переменных, где $Q$ - содержащий начало координат компакт в $\mathbb{R}^{n}$, а также в некоторых его подпространствах (Берлинга и Румье; см., например, монографию [9, гл. 1, §§ 1, 5], а также статью [10] и библиограбию к ней). В частности, при $n=2$ проблема Бореля ставится так: найти функцию $v\left(x_{1}, x_{2}\right)$ из $C_{(\mathbb{R})}^{\infty}(Q)$ такую, что

$$
\left.\frac{\partial^{\alpha+\beta} v\left(x_{1}, x_{2}\right)}{\partial x_{1}^{\alpha} \partial x_{2}^{\beta}}\right|_{x_{1}=x_{2}=0}=d_{\alpha, \beta}, \quad \alpha, \beta=0,1,2, \ldots
$$

Здесь $\left\{d_{\alpha, \beta}: \alpha, \beta=0,1,2, \ldots\right\}$ - произвольно заданная система вещественных чисел.

Что же касается аналитических решений многомерной задачи Бореля, то, насколько нам известно, результатов в этом направлении получено не было в связи с тем, что методы, примененные Борелем и его последователями в одномерной ситуации, оказались неэффективными в многомерном случае (при рассмотрении аналитических решений). Метод данной работы позволяет восполнить этот пробел.

Для простоты изложения ограничимся двумерной проблемой $(n=2)$ и будем искать решение в виде ряда экспонент. Итак, рассматривается задача

$$
\left.\frac{\partial^{\alpha+\beta} v}{\partial z_{1}^{\alpha} \partial z_{2}^{\beta}}\right|_{z_{1}=z_{2}=0}=d_{\alpha, \beta}, \quad \alpha, \beta=0,1,2, \ldots
$$

Решение этой задачи ищем в виде ряда

$$
v\left(z_{1}, z_{2}\right)=\sum_{k=1}^{\infty} c_{k} \exp \left(\lambda_{k, 1} z_{1}+\lambda_{k, 2} z_{2}\right)
$$

Показатели $\lambda_{k}$ (т.е. $\lambda_{k, 1}, \lambda_{k, 2}$ ) выбираем следующим образом:

$$
\begin{gathered}
\forall k \geqslant 1 \quad \lambda_{k, 1}=\rho_{k}, \quad \lambda_{k, 2}=\tau_{k} \rho_{k} ; \quad \tau_{k}>0, \rho_{k}>0 ; \\
\lim _{k \rightarrow \infty} \tau_{k}=\lim _{k \rightarrow \infty} \rho_{k}=\infty ; \quad \lim _{k \rightarrow \infty} \frac{\ln \tau_{k}}{\ln \rho_{k}}=0 .
\end{gathered}
$$


Рассмотрим вспомогательную систему

$$
\sum_{k=1}^{\infty} x_{k}\left(\lambda_{k, 1}\right)^{\alpha}\left(\lambda_{k, 2}\right)^{\beta}=d_{\alpha, \beta}, \quad \alpha, \beta=0,1,2, \ldots
$$

Для того чтобы использовать вышеприведенную теорему Полиа (которая имеет одномерный характер), необходимо перенумеровать все пары индексов $(\alpha, \beta)$, где $\alpha \geqslant 0$, $\beta \geqslant 0$, в виде обычной последовательности $0,1,2, \ldots$. Такую нумерацию осуществим следуюшим образом: будем нумеровать пары в порядке возрастания величины $\alpha+\beta$, а в каждой групше пар с одинаковой суммой $\alpha+\beta$ в порядке возрастания индекса $\beta$ : $(0,0),(1,0),(0,1),(2,0),(1,1),(0,2), \ldots$. После такой перенумерации система $(21)$ примет вид

$$
\sum_{k=1}^{\infty} x_{k} \mu_{k, n}=d_{n}, \quad n=0,1,2, \ldots
$$

Зафиксируем какое-либо $k \geqslant 1$ и возьмем произвольное $s \geqslant 1$. Пусть сначала $s$ - номер пары вида $(m+1,0)$. Тогда

$$
\begin{aligned}
\sum_{j=1}^{s-1}\left|\mu_{k, j}\right| & =\sum_{r=0}^{m} \sum_{\alpha+\beta=r}\left(\lambda_{k, 1}\right)^{\alpha}\left(\lambda_{k, 2}\right)^{\beta}=\sum_{r=0}^{m}\left(\rho_{k}\right)^{r} \sum_{\beta=0}^{r}\left(\tau_{k}\right)^{\beta} \\
& <\frac{\tau_{k}^{m+1}-1}{\tau_{k}-1} \cdot \frac{\rho_{k}^{m+1}-1}{\rho_{k}-1} .
\end{aligned}
$$

Так как в силу (20) вьполняется равенство

$$
0=\lim _{k \rightarrow \infty} \frac{\tau_{k}^{m}}{\rho_{k}}=\lim _{k \rightarrow \infty} \frac{\left(\tau_{k}^{m+1}-1\right)\left(\rho_{k}^{m+1}-1\right)}{\left(\tau_{k}-1\right)\left(\rho_{k}-1\right) \rho_{k}^{m+1}}
$$

то

$$
\varlimsup_{k \rightarrow \infty} \frac{1}{\left|\mu_{k, s}\right|} \sum_{j=1}^{s-1}\left|\mu_{k, j}\right| \leqslant 0
$$

т.е.

$$
\varlimsup_{k \rightarrow \infty} \frac{1}{\left|\mu_{k, s}\right|} \sum_{j=1}^{s-1} \mu_{k, j}=0, \quad s=2,3, \ldots
$$

Пусть теперь $s$ - номер пары $(m+1, r)$, где $0<r \leqslant m+1$. Тогда

$$
\sum_{j=1}^{s-1} \mu_{k, j}=\sum_{r=0}^{m} \sum_{\alpha+\beta=r}\left(\lambda_{k, 1}\right)^{\alpha}\left(\lambda_{k, 2}\right)^{\beta}+\rho_{k}^{m+1} \sum_{j=0}^{r-1} r_{k}^{j}, \quad \mu_{k, s}=\tau_{k}^{r} \rho_{k}^{m+1} .
$$

Возьмем $k$ настолько большим, чтобы $\tau_{k} \geqslant 1, \rho_{k} \geqslant 1$. Имеем

$$
\frac{1}{\mu_{k, s}} \sum_{j=1}^{s-1} \mu_{k, j} \leqslant \frac{1}{\rho_{k}^{m+1}} \sum_{r=0}^{m} \sum_{\alpha+\beta=r}\left(\lambda_{k, 1}\right)^{\alpha}\left(\lambda_{k, 2}\right)^{\beta}+\frac{1}{\tau_{k}^{r}} \sum_{j=0}^{r-1} \tau_{k}^{j} .
$$


Первое слагаемое в правой части неравенства $(22)$ при $k \rightarrow \infty$ стремится к нулю, как только что было показано. Далее,

$$
\frac{1}{\tau_{k}^{r}} \sum_{j=0}^{r-1} \tau_{k}^{j}=\frac{\tau_{k}^{r}-1}{\tau_{k}^{r}\left(\tau_{k}-1\right)}<\varepsilon \quad \forall k \geqslant K_{0}(\varepsilon)
$$

Следовательно,

$$
\varlimsup_{k \rightarrow \infty} \frac{1}{\mu_{k, s}} \sum_{j=1}^{s-1} \mu_{k, j} \leqslant 0, \quad \lim _{k \rightarrow \infty} \frac{1}{\mu_{k, s}} \sum_{j=1}^{s-1} \mu_{k, j}=0
$$

На основании теоремы Полиа система (21) имеет бесконечное множество решений $\left\{c_{n}\right\}_{n=1}^{\infty}$ таких, что

$$
\sum_{k=1}^{\infty}\left|c_{k}\right| \cdot\left|\lambda_{k, 1}\right|^{\alpha} \cdot\left|\lambda_{k, 2}\right|^{\beta}<\infty, \quad \alpha, \beta=0,1,2, \ldots
$$

Положим $\Pi_{j}=\left\{z_{j}: \operatorname{Re} z_{j}<0\right\}, \bar{\Pi}_{j}=\left\{z_{j}: \operatorname{Re} z_{j} \leqslant 0\right\}, \Gamma_{j}=\left\{z_{j}: \operatorname{Re} z_{j}=0\right\}$, $j=1,2 ; \Pi=\Pi_{1} \times \Pi_{2}, \bar{\Pi}=\bar{\Pi}_{1} \times \bar{\Pi}_{2}, \Gamma=\left(\Gamma_{1} \times \bar{\Pi}_{2}\right) \cup\left(\bar{\Pi}_{1} \times \Gamma_{2}\right)$.

Из соотношений (23) следует, что ряд (19) сходится абсолютно в $C_{b}^{\infty}(\bar{\Pi})$. Здесь $C_{b}^{\infty}(\bar{\Pi})$ определяется как пространство всех функций $v\left(z_{1}, z_{2}\right)$, непрерьвных на $\bar{\Pi}$, имеющих непрерьвные частные производные всех порядков по $z_{1}$ и $z_{2}$ и ограниченных на $\bar{\Pi}$ вместе с любой такой производной. Топология в $C_{b}^{\infty}(\bar{\Pi})$ задается набором норм

$$
\left|y_{n}\right|=\sup \left\{\left|\frac{\partial^{\alpha+\beta} y}{\partial z_{1}^{\alpha} \partial z_{2}^{\beta}}\right|:\left(z_{1}, z_{2}\right) \in \bar{\Pi}, \alpha \geqslant 0, \beta \geqslant 0, \alpha+\beta \leqslant n\right\}, \quad n=0,1, \ldots
$$

Отсюда нетрудно вывести, что $v\left(z_{1}, z_{2}\right) \in C_{b}^{\infty}(\bar{\Pi})$; при этом функция $v$ аналитична в П и условия (18) выполняются. Сформулируем полученный результат, используя понятие $B$-множества, которое вводится так же, как в одномерном случае.

Теорема 6. Множество $\bar{\Pi}$ - В-множество; для любой системы комплексных чисел $\left\{d_{\alpha, \beta}: \alpha, \beta=0,1,2, \ldots\right\}$ существует решение задачи (18) из $C_{b}^{\infty}(\bar{\Pi})$, представимое в виде ряда (19), коэффиииенты $c_{k}$ которого определяются из системы (21), а показатели $\lambda_{k, j}(j=1,2)$ - из соотношения (20).

СлЕДСТВИЕ. Пусть $\bar{D}=\bar{D}_{1} \times \bar{D}_{2}$, әде $D_{j}, j=1,2,-$ односвязная область в $\mathbb{C}$ с замкнутой жсордановой граничей $\Gamma_{j}$ класса $C^{\infty}$, содержащей точку $z_{j}=0$. Тогда $\bar{D}-$ В-множество, т.е. для любой системы комплексных чисел $\left\{d_{\alpha, \beta}\right\}_{\alpha, \beta=0}^{\infty}$ существует решение задачи (18) из $C_{b}^{\infty}(\bar{D})$.

Аналогично можно получить двумерные (и многомерные) аналоги других результатов данной статьи, но мы уже не будем здесь на этом останавливаться. 


\section{СПИСОК ЦИТИРОВАННОЙ ЛИТЕРАТУРЫ}

[1] Borel E. Sur quelques points de la theorie des fonctions // Ann. Sci. Ecole Norm. 1895. V. $12(3)$. P. 9-55.

[2] Митягин Б.С. О бесконечно дифференцируемой функции с заданньми значениями производных в точке // Докл. АН СССР. 1961. Т. 138. № 2. С. 289-292.

[3] Petzsche H.-J. On E. Borel's theorem // Math. Ann. 1988. V. 282. P. 299-313.

[4] Cooke R. G. Infinite matrices and sequence spaces. London, 1950.

[5] Бибербах Л. Аналитическое продолжение. М.: Наука, ГИФМЛ, 1967.

[6] Polya G. Eine Verallegemeinerung des Fabryschen Lückensatzes // Nachr. Gesellsch. Wissensch. Göttingen. 1927. P. 187-195.

[7] Valiron G. Sur les solutions des équations différentielles linéares d'ordre infini et à coefficients constants // Ann. Ec. Norm. Sup. 1929. V. 46. P. 25-53.

[8] Коробейник Ю.Ф. О задаче Коши для линейного дифференциального уравнения бесконечного порядка // Литовск. матем. сб. (Lietuvos Matematikos Rinkiny). 1965. Т. 5. № 3. C. $397-420$.

[9] Нарасимхан Р. Анализ на действительных комплексных многообразиях. М.: Мир, 1971.

[10] Meise R., Taylor B. A. Whitney's extension theorem for ultra-differentiable functions of Beurling type // Ark. Mat. 1988. V. 26. P. 265-287.

Ростовский государственный университет

Поступило

E-mail: kor@math.rsu.ru

29.05 .1998

Исправленный вариант

20.05.1999 